04

\title{
Параметрическое исследование двух устойчивых форм горения разряда в холловском двигателе
}

\author{
(С) И.А. Хмелевской, ${ }^{1,2}$ Д.А. Томилин ${ }^{1}$ \\ ${ }^{1}$ Исследовательский центр им. М.В. Келдыша, \\ 125438 Москва, Россия \\ ${ }^{2}$ Московский фризико-технический институт (государственный университет), \\ 141701 Долгопрудный, Московская обл., Россия \\ e-mail: khmelevskoi@kerc.msk.ru
}

Поступило в Редакцию 22 января 2019 г.

В окончательной редакции 22 января 2019 г.

Принято к публикации 25 марта 2019 г.

\begin{abstract}
Экспериментально исследовано поведение интегральных параметров работы холловского двигателя (тяга, удельный импульс тяги) в двух устойчивых режимах горения разряда, существенно отличающихся друг от друга по анодному КПД. Исследования проводились на лабораторной модели холловского двигателя с выдвинутым слоем со средним диаметром разрядного канала $77 \mathrm{~mm}$ в диапазоне напряжения разряда 500-900 V и расходе газа от 2 до $5 \mathrm{mg} / \mathrm{s}$. Наиболее яркими отличительными признаками наблюдаемых режимов горения являются форма плазменной струи и значение тока разряда при практически идентичных входных параметрах (расход газа, напряжение разряда, величина магнитного поля). Показано, что во всем исследуемом диапазоне входных параметров при перестроении из оптимального режима (с точки зрения КПД) в неоптимальный основные интегральные характеристики двигателя претерпевают скачкообразное изменение: возрастает ток разряда на $10-30 \%$ с одновременным относительным падением тяги на 5-15\% и КПД на 20-40\%. Детальное исследование структуры анодного КПД показало, что при перестроении скачком изменяется эффективность использования электронного тока (отношение тока ионов к току разряда), т. е. электронная проводимость в разрядном канале двигателя.
\end{abstract}

Ключевые слова: электроракетные двигатели, холловские двигатели, параметры эффективности, устойчивость горения разряда, режимы горения разряда.

DOI: $10.21883 /$ JTF.2019.09.48061.20-19

\section{Введение}

Холловский двигатель (ХД) - разновидность электрических ракетных двигателей - разрабатывается с середины XX века и используется для коррекции орбиты и ориентации космических аппаратов (КА). Детальное описание и принцип работы двигателя можно найти в работах $[1,2]$.

Тенденции развития современных КА связаны с увеличением срока их активного существования и повышения относительной доли полезной нагрузки, что приводит к необходимости разработки двигателей с повышенным удельным импульсом тяги (свыше $2000 \mathrm{~s}$ ), что, как правило, достигается увеличением разрядного напряжения. Обеспечение длительного ресурса, стабильной и устойчивой работы являются ключевыми задачами при создании таких двигателей.

В последнее время активно развивается направление создания ХД с выдвинутым слоем, в которых организуется такая геометрия магнитной системы и конструктивных элементов двигателя, при которой практически отсутствует типичная для двигателей классической схемы ХД эрозия выходной части разрядного канала. Достигается такой эффект выдвижением максимума магнитного поля на среднем диаметре разрядного канала вперед по потоку за срез выходной плоскости двигателя с соответствующим подбором формы профилей выходных изоляторов под конфигурацию магнитных силовых линий, при котором поверхность изоляторов имеет форму расширяющегося конуса, а магнитные силовые линии практически параллельны этой поверхности. В западной литературе подобный способ организации конструктивных элементов двигателя называется „магнитным экранированием“ („magnetic shielding“) [3,4]. Такой метод организации конструкции позволяет выдвинуть вперед по потоку плазменный слой (область разряда двигателя, содержащая основное электрическое поле), и, таким образом, существенно снизить уровень эрозии выходных изоляторов и соответственно увеличить ресурс работы двигателя, в том числе при высоких значениях разрядного напряжения.

Свойство плазменного слоя в ХД „привязываться“ к максимуму магнитного поля на средней линии разрядного канала отмечено еще в ранних разработках советского периода [5], а работа по созданию двигателей с выдвинутым слоем в 80-90-х годах прошлого века привела к созданию разнообразных конструкций ХД, в которых этот принцип был реализован [6,7], и тем не менее множество вопросов остается до сих пор открытыми. При выдвижении слоя вперед по потоку 
могут существенно изменяться физические процессы, происходящие в слое, в частности, снижение уровня взаимодействия плазменного потока со стенками разрядного канала может приводить к снижению уровня электронной проводимости, и, как следствие, к развитию того или иного типа плазменных неустойчивостей, что, в свою очередь, может спровоцировать перестроение режима горения разряда, при котором удельные характеристики двигателя будут так же претерпевать существенные изменения.

Подобные перестроения наблюдались в ряде работ [8-10], однако заметим, что этот процесс имеет место не только для конструкций с выдвинутым слоем [11-16]. Опыт разработки двигателей различных типоразмеров в ГНЦ ФГУП „Центр Келдыша“ показывает, что перестроения могут происходить при уменьшении расхода газа при фиксированном магнитном поле, либо при увеличении магнитного поля при постоянном расходе газа $[15]$, также перестроения наблюдались при расширении выходной части разрядной камеры вследствие эрозии в процессе выработки ресурса двигателя [16]. Публикаций, посвященных систематическому изучению данной тематики крайне мало, поэтому отсутствует строгая классификация наблюдаемых режимов горения разряда ХД и условий, при которых происходит переход из одного режима в другой. Далее для определенности будем называть неоптимальный с точки зрения КПД режим горения разряда „колокол“, а оптимальный „спица“, названия выбраны исключительно ассоциативно с формой струи двигателя, которая в разных режимах горения заметно отличается.

Целью настоящей работы было экспериментальное исследование поведения интегральных параметров работы двигателя (ток разряда, тяга, удельный импульс тяги, КПД) в зависимости от режима горения разряда при различных значениях напряжения и мощности разряда, исследование эффективности процессов образования и ускорения ионов в двигателе путем анализа структуры анодного КПД с использованием экспериментальных данных об основных выходных характеристиках двигателя и параметрах плазменной струи.

\section{Экспериментальная установка и условия проведения испытаний}

Испытания проводились в ГНЦ ФГУП „Центр Келдыша“ в вакуумной камере КВУ-90 диаметром $3.8 \mathrm{~m}$ и длиной $8 \mathrm{~m}$. Давление в камере в процессе испытаний поддерживалось при помощи криогенных вакуумных насосов на уровне не выше $5 \cdot 10^{-3}$ Ра. Вакуумная установка оснащена системой зондовой диагностики, включающей в себя трехсеточные зонды с задерживающим потенциалом, позволяющей проводить измерения угловой расходимости и энергетического состава ионного потока струи двигателя. Контроль работы двигателя и

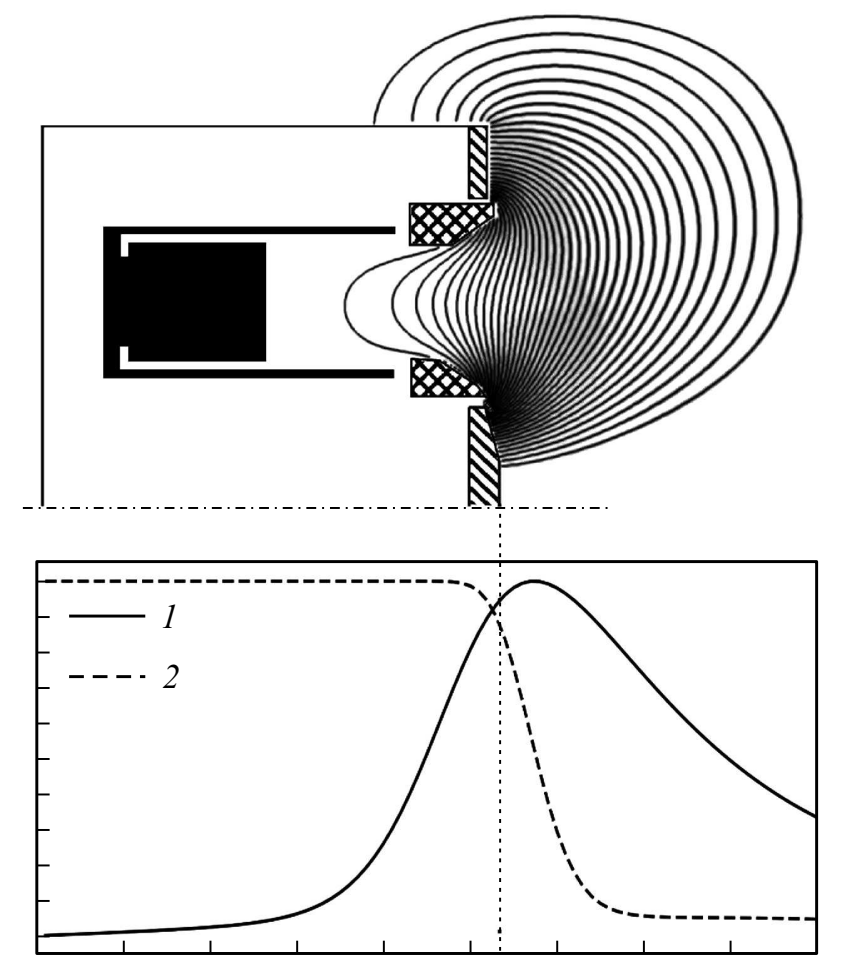

Рис. 1. Схематическое изображение двигателя с силовыми линиями магнитного поля. Косая штриховка - магнитные полюса двигателя, перекрестная штриховка - керамические выходные изоляторы двигателя, черная заливка - анодный блок, 1 - характерное распределение магнитного поля на среднем диаметре разрядного канала, 2 - характерное распределение потенциала на среднем диаметре разрядного канала.

его параметров осуществляется автоматизированной системой управления и сбора данных, включающих в себя токи, потенциалы, расход газа, температуры элементов двигателя и показания тягоизмерительного устройства (ТИУ).

Для проведения исследования использовался ХД со средним диаметром разрядного канала $77 \mathrm{~mm}$ с выдвинутым слоем (рис. 1). Особенностью конструкции данного двигателя является вынесение максимума магнитного поля на среднем диаметре двигателя за точку пересечения оси двигателя и выходной плоскости, проходящей через его полюса, т. е. вперед по потоку. Выходная часть разрядного канала двигателя выполнена таким образом, что ее рабочая поверхность практически параллельна магнитным силовым линиям вблизи ее. Двигатель был дополнительно оснащен набором термопар на всех основных конструктивных узлах, в том числе на внутреннем и наружном выходных изоляторах, непосредственно контактирующих с плазменным слоем.

Расход газа в эксперименте варьировался в диапазоне от 2 до $4 \mathrm{mg} / \mathrm{s}$ с шагом $0.5 \mathrm{mg} / \mathrm{s}$, напряжение разряда изменялось в диапазоне от 500 до $900 \mathrm{~V}$ с шагом $100 \mathrm{~V}$. При каждом значении расхода газа и напряжения разряда путем изменения величины магнитного поля осу- 

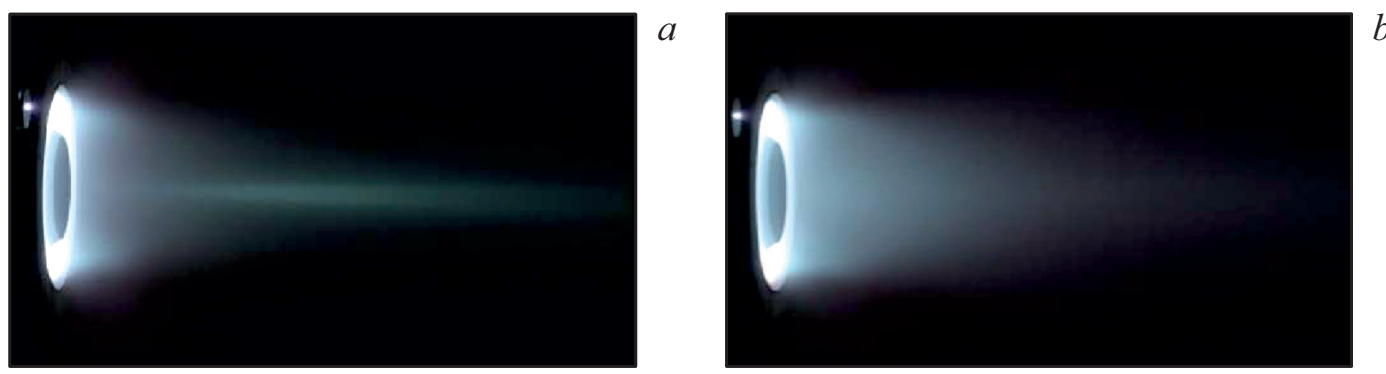

Рис. 2. Внешний вид плазменной струи ХД в режиме „спица“ $(a)$ и режиме „колокол“ $(b)$.

ществлялся поиск точки перехода из режима в режим, после этого производилось измерение параметров двигателя, включая параметры струи, в обоих режимах вблизи этой точки путем незначительного изменения магнитного поля, позволяющего спровоцировать работу двигателя либо в режиме „спица“, либо в режиме „колокол“. Перестроения из режима „спица“ в режим „колокол“ достигались увеличением магнитного поля, обратное перестроение достигалось соответственно его уменьшением относительно точки перехода. Отметим, что перестроения происходили с некоторым „гистерезисом“6 по магнитному полю, т.е. перестроение из „спицы“ в „колокол“ происходило при несколько большем значении поля, чем обратное перестроение. Также заметим, что изначально исследование проводилось в диапазоне от 300 до $1000 \mathrm{~V}$, однако до $500 \mathrm{~V}$ спровоцировать перестроение не удавалось, и двигатель работал все время в режиме „спица“, а свыше $900 \mathrm{~V}$ перегрев конструкции в режиме „колокол“ становился настолько существенным, что не удавалось достигнуть устойчивой стационарной работы, поэтому далее результаты в основном приведены только для диапазона напряжений 500-900 V.

\section{Результаты испытаний}

На рис. 2 представлен внешний вид струи ХД при режимах горения „спица“ и „колокол“. Из внешних визуальных признаков режима „спица“ можно отметить ярко выраженную плазменную „спицу“, располагающуюся на оси двигателя, в струе в целом преобладают зеленоватобелые оттенки; в режиме „колокол“ плазменная „спица“ пропадает, форма струи напоминает трубу или колокол, в струе преобладают красно-белые оттенки.

На рис. 3 представлена зависимость точки перехода из режима в режим по магнитному полю от напряжения разряда, разные кривые соответствуют различным значениям расхода газа. Каждая кривая делит область на две части: все точки выше кривой соответствуют работе двигателя в режиме „колокол“, все точки ниже кривой - работе в режиме „спица“. Как будет показано дальше, такое деление равносильно указанию рабочего диапазона двигателя, так как режим „колокол“, особенно при высоком напряжении разряда, является неприемлемым для длительной работы.

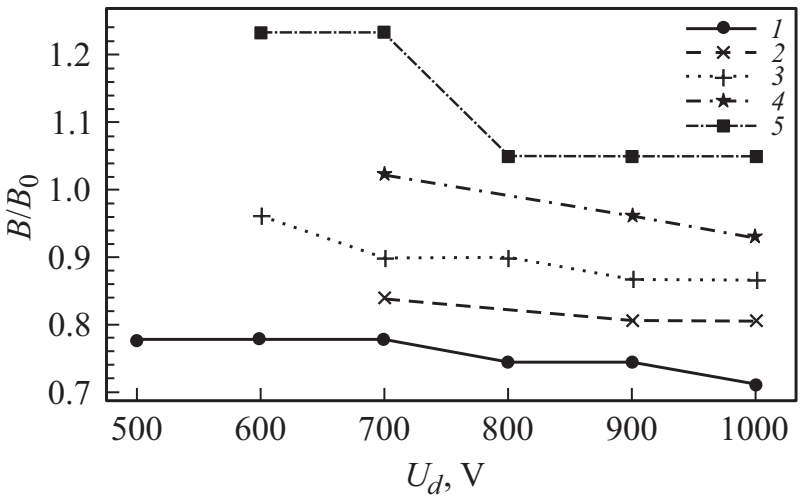

Рис. 3. Относительная величина магнитного поля, при котором происходит перестроение из одного режима в другой, в зависимости от напряжения разряда при различных значениях расхода газа: $1-2,2-2.5,3-3,4-3.5,5-4 \mathrm{mg} / \mathrm{s}$.

Из анализа рис. 3 можно выделить две отчетливые тенденции: диапазон работы по магнитному полю в режиме „спица“ расширяется при увеличении расхода газа и сужается при увеличении напряжения разряда. Здесь отметим, что граница рабочего диапазона снизу по магнитному полю также присутствует, однако она практически не зависит от расхода и несколько увеличивается с ростом напряжения разряда.

Далее приведем сравнение характеристик работы двигателя в режиме „спица“ и „колокол“. Данные приведены только для расхода газа $2 \mathrm{mg} / \mathrm{s}$, при более высоких значениях расхода газа качественно все тенденции сохраняются. На рис. 4 отражены основные параметры работы ХД. При перестроении из режима „спица“ в режим „колокол“ при постоянном значении расхода газа происходит увеличение тока разряда на $10-30 \%$ (рис. $3, a$ ) с одновременным уменьшением тяги на 5-15\% (рис. $4, c$ ), КПД двигателя уменьшается на $20-40 \%$ в относительных единицах (рис. $4, d$ ). С увеличением напряжения разряда негативные последствия перестроения становятся критичнее: разница в токе разряда, тяге и КПД между режимом „спица“ и режимом „колокол“ увеличивается.

На рис. 5 приведено поведение температур внутреннего и наружного выходных изоляторов, которые находятся в непосредственном контакте с плазменным 

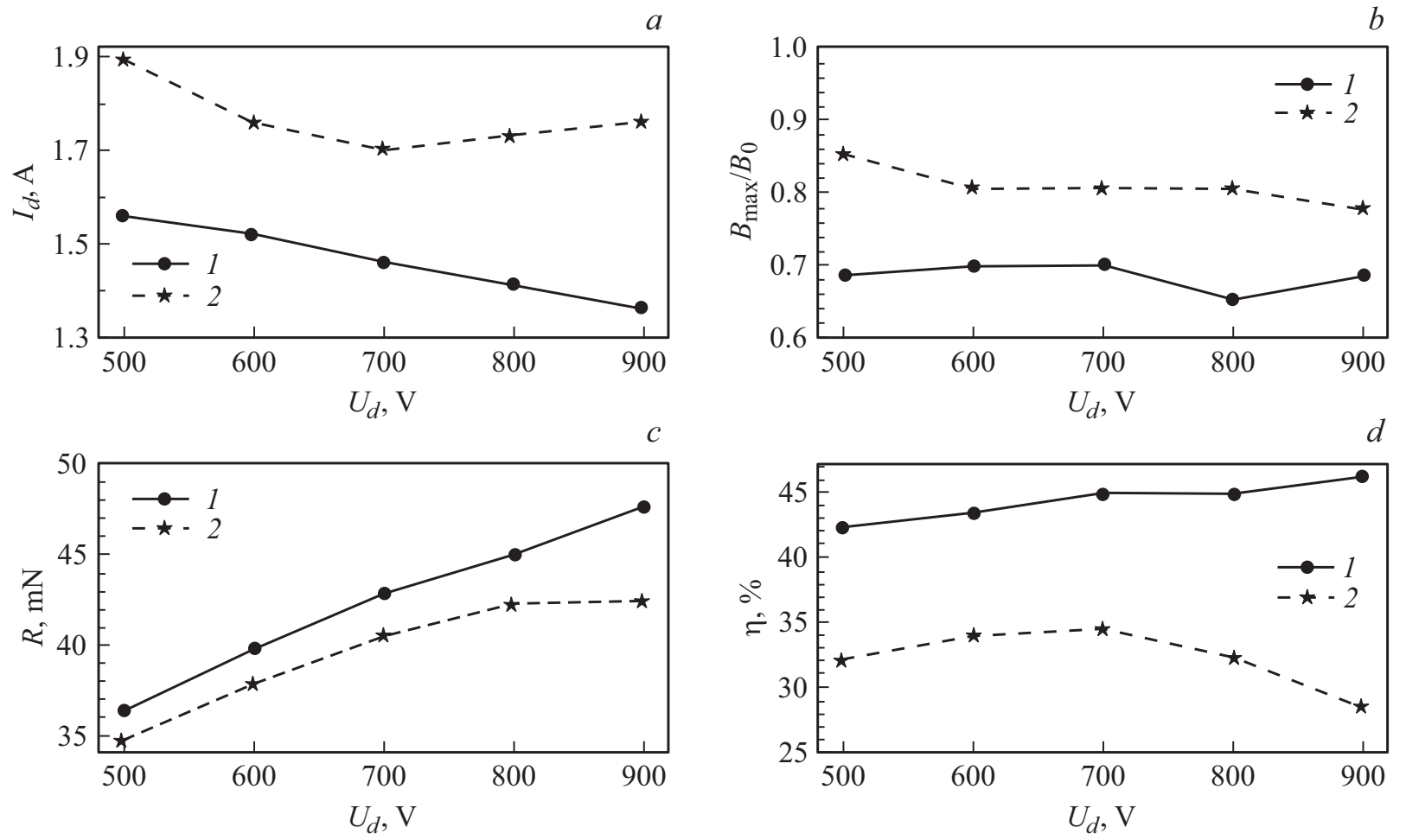

Рис. 4. Основные параметры работы в режимах „спица“ и „колокол“: $a-$ ток разряда, $b-$ относительная величина магнитного поля, $c$ - тяга, $d-$ анодный КПД, \%. 1 - „спица“, 2 - „колокол“.
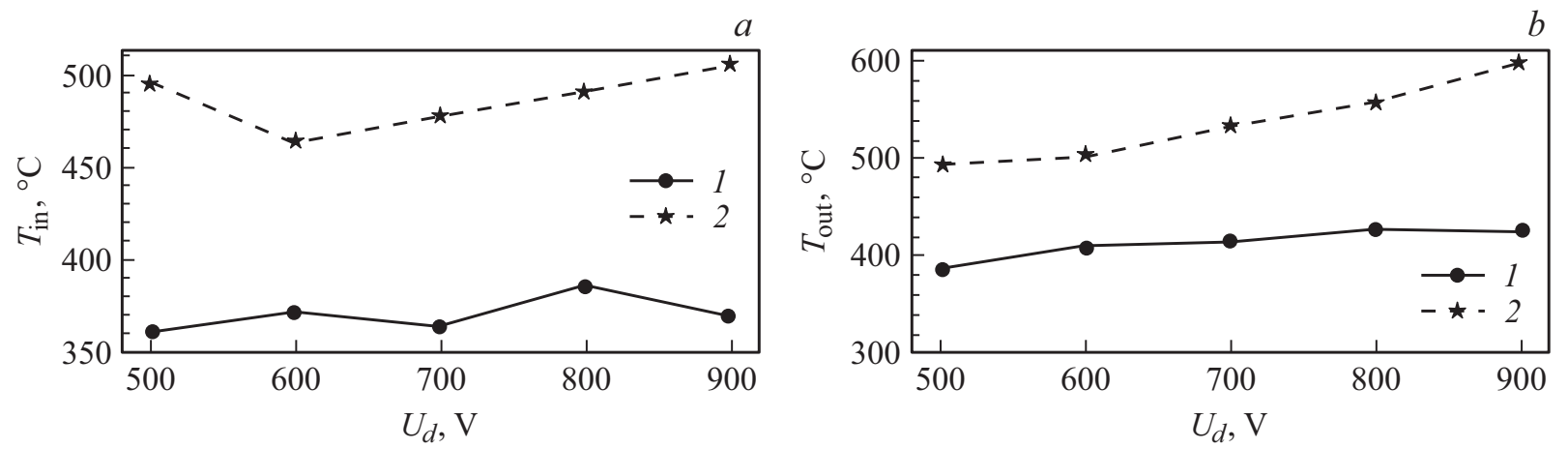

Рис. 5. Температура внутреннего $(a)$ и наружного $(b)$ выходных керамических изоляторов двигателя в режимах „спица“ и „колокол““ в зависимости от напряжения разряда. 1 - „спица“, 2 - „колокол““.

слоем разряда двигателя, т. е. испытывают максимальные термические нагрузки.

При перестроении между режимами температура колец отличается приблизительно на $100-150^{\circ} \mathrm{C}$, следовательно, тепловой поток на выходные кольца в режиме „колокол“ существенно больше, чем в режиме „спица“. При большей мощности разряда в режиме „колокол“ эта разница в тепловых потоках приводит к сильному перегреву конструктивных элементов двигателя.

На основе анализа параметров работы можно однозначно сказать, что оптимальным режимом работы ХД является режим „спица“, а режим „колокол“ является неприемлемым.
Для более детального изучения процессов, протекающих в том и другом режиме, была исследована структура анодного КПД двигателя при помощи методики, изложенной в [17]. В основе методики лежит представление основных интегральных параметров работы ХД в виде произведения набора коэффициентов эффективности $R=\eta_{m} \gamma \sqrt{\frac{2 m_{e} U_{d}}{e}}, \eta=\eta_{m} \eta_{l} \gamma^{2}$, где $\eta_{m}-$ коэффициент использования рабочего тела (отношение массового тока ионов к расходу газа), $\eta_{I}$ - коэффициент эффективности использования электронного тока (отношение тока ионов к току разряда), $\gamma^{2}=\gamma_{E} \gamma_{\theta}$, где $\gamma_{\theta}$ - описывает снижение тяги из-за углового разброса скоростей ионов, $\gamma_{E}$ - показывает снижение тяги из-за 

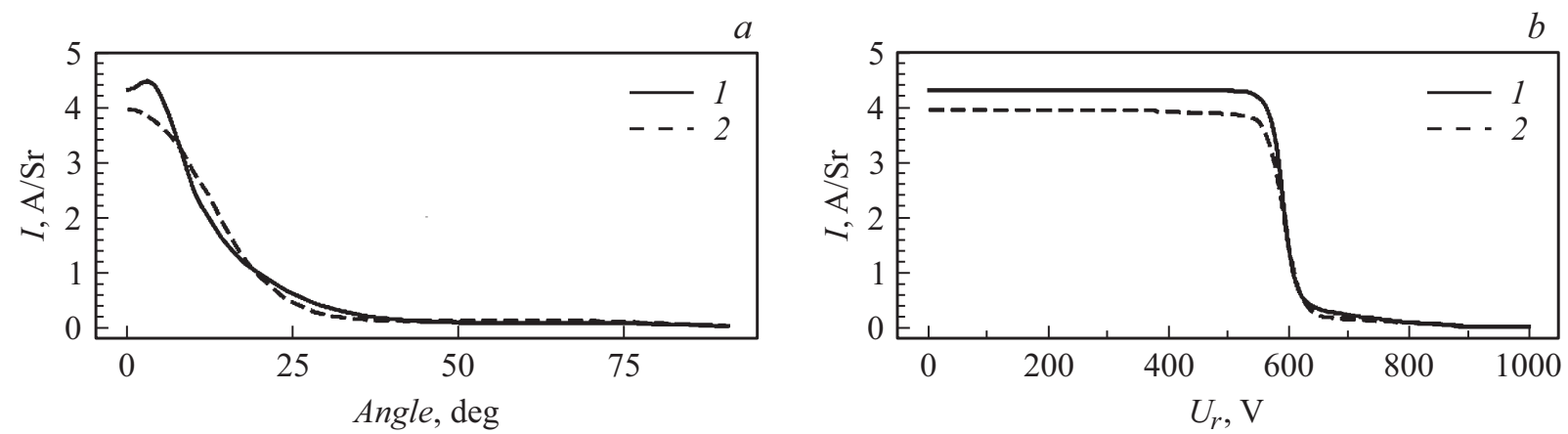

Рис. 6. Угловое распределение в режимах „спица“ и „колокол“ для напряжения разряда 600 V. 1 - „спица“, 2 - „колокол“.
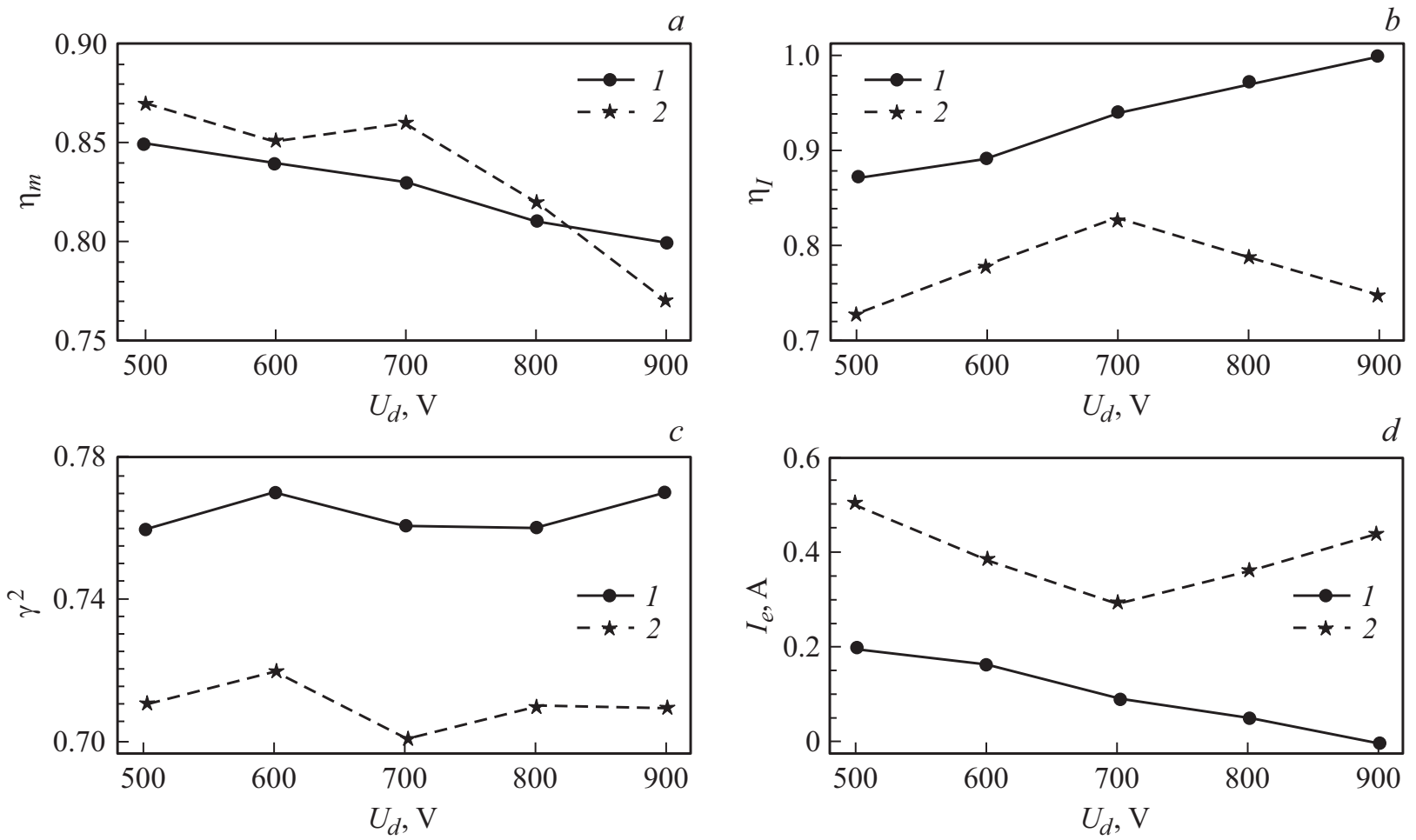

Рис. 7. Параметры струи в режимах „спица“ и „колокол“: $a-\eta_{m}, b-\eta_{I}, c-\gamma^{2}, d-$ электронная добавка тока. $1-$ „спица“, $2-$ „колокол“.

энергетического распределения ионов. Вычисление данных коэффициентов требует знания об энергетическом и угловом распределении в ионном потоке, для чего использовалась система зондовой диагностики на основе трехсеточного зонда с задерживающим потенциалом.

Пример углового и энергетического распределений для обоих режимов горения для напряжения разряда $600 \mathrm{~V}$ и расходе газа $2 \mathrm{mg} / \mathrm{s}$ приведен на рис. 6.

Рассчитанные параметры эффективности в зависимости от напряжения разряда в различных режимах приведены на рис. 7, $a-c$; на рис. 7, $d$ приведена зависимость электронной добавки тока (разница между током разряда и током ионов).

Из анализа графиков на рис. 7 можно сделать вывод, что коэффициент использования рабочего тела в обоих режимах приблизительно одинаков, отличие составляет всего несколько процентов, в то время как эффективность использования электронного тока в режиме „спица“ в среднем более чем на $10 \%$ выше. Также в этом режиме лучше угловое и энергетическое распределение, $\gamma^{2}$ выше приблизительно на 5-6\%. Небольшое отличие в коэффициенте использования рабочего тела и сильное отличие в эффективности использования электронного тока говорит о том, что ток разряда в режиме „колокол“ растет в основном за счет электронной составляющей тока. Из рис. 7, $d$ видно, что электронная добавка для напряжений разряда $500-700 \mathrm{~V}$ в $\sim 1.5$ раза выше в режиме „колокол“, а для 800-900 V электронная добавка тока в режиме „колокол“ более чем в 5 раз превышает аналогичную величину в режиме „спица“. 


\section{Обсуждение полученных результатов}

Проведенные испытания показали, что при напряжении разряда свыше $500 \mathrm{~V}$ при относительно низких значениях расхода газа и достаточно высоком магнитном поле происходит скачкообразное перестроение режима работы ХД. При перестроении тяга падает на 5-15\%, ток разряда при постоянном расходе газа возрастает на $10-30 \%$, что приводит к существенному падению КПД двигателя. Температура конструктивных элементов двигателя возрастает на 20-40\%, при этом проведенные ранее исследования [15] показывают, что плазменный слой в режиме, аналогичном режиму „колокол“, расположен ближе к аноду по сравнению с режимом „спица“, что увеличивает тепловой поток на рабочие поверхности двигателя и объясняет резкий рост температуры изоляторов, наблюдавшийся в настоящей работе. Данный процесс приводит, с одной стороны, к росту тепловой напряженности конструкции и сокращению ресурса конструктивных элементов, в частности, это касается обмоток магнитных катушек, ресурс которых зависит от теплового состояния. С другой стороны, смещение слоя к аноду и возрастание потока плазмы на поверхность выходных изоляторов приводит к повышенному уровню скорости эрозии. Все это в совокупности позволяет сделать однозначный вывод о том, что режим „колокол“ является неприемлемым для работы ХД.

Однако наряду с последним выводом можно утверждать, что перестроение из режима „спица“ в режим „колокол“ может быть парировано либо увеличением расхода газа, т. е. мощности разряда, либо уменьшением величины магнитного поля. Важность этого замечания обусловлена тем, что в современных методиках масштабирования ХД [18] при выборе геометрии двигателя обычно оперируют только мощностью разряда, не учитывая то, при каком напряжении разряда эта мощность получена. В данной работе было показано, что диапазон устойчивой и эффективной работы по магнитному полю при постоянной мощности с ростом напряжения разряда быстро сокращается из-за перестроения режима горения разряда, т. е. выбор напряжения при заданной мощности и фиксированной геометрии двигателя не может быть произвольным.

Наблюдаемые перестроения имеют место на двигателях разных конструкций, поэтому можно предположить, что причиной перестроения является какой-то фундаментальный физический процесс, не связанный явно с конструктивными особенностями и режимом работы ХД, например, процесс проводимости электронов за срезом разрядного канала. Качественно причина перестроения из „спицы“ в „колокол“ может заключаться в уровне электронной проводимости, необходимом для поддержания устойчивого горения разряда в режиме „спица“. При фиксированном напряжении разряда перестроение из „спицы“ в „колокол“ происходит либо при увеличении значения магнитного поля, либо при уменьшении расхода газа, т. е. при уменьшении проводимости по всему плазменному объему, при перестроении скачком в несколько раз возрастает именно электронная добавка тока (рис. 7,d). Обратный процесс может быть спровоцирован либо уменьшением магнитного поля, либо увеличением расхода с небольшим „гистерезисом“, тем не менее в обоих случаях в канале увеличивается уровень проводимости электронов. Таким образом, можно предположить, что эффект перестроения связан с развитием какого-то процесса, который стремится поддерживать неразрывность электронной компоненты тока.

Для построения более детальной картины различий двух наблюдаемых режимов горения разряда и причин перестроений между ними необходимо разобраться, с каким типом проводимости происходят скачкообразные изменения при перестроении. Вопрос о проводимости электронов в разряде ХД остается до сих пор открытым: классического столкновительного механизма переноса на тяжелых частицах и величины пристеночной проводимости оказывается недостаточно для полного описания величины электронного тока. Существенное влияние оказывает так называемая аномальная проводимость, которую часто связывают с колебаниями и волнами в разряде ХД [19]. Так как расход газа при перестроении между режимами был постоянным, а магнитное поле отличалось незначительно, то можно считать, что величина классического переноса электронов была приблизительно одинакова в обоих режимах горения. Таким образом, прирост электронной проводимости при перестроении может происходить за счет либо скачкообразного роста аномального переноса, либо пристеночной проводимости, либо сложной их комбинации, в любом случае это должно отразиться на спектре осцилляций в плазменном объеме. Подобного рода исследование станет предметом дальнейших работ в этом направлении.

\section{Заключение}

В ГНЦ ФГУП „Центр Келдыша“ проведено параметрическое исследование двух режимов горения разряда в двигателе холловского типа. Проведено сравнительное исследование основных интегральных параметров работы двигателя в обоих режимах, а также изучена структура коэффициентов эффективности анодного КПД. При перестроении двигателя из режима „спица“ в режим „колокол“ при неизменном значении напряжения разряда и расхода газа резко увеличивается ток разряда с одновременным уменьшением тяги и существенным снижением КПД двигателя. С точки зрения деградации интегральных параметров работы двигателя и роста температуры его конструктивных элементов перестроение из „спицы“ в „колокол“ можно назвать катастрофическим.

Показано, что определяющим отличием двух форм горения разряда является значение электронной добавки 
тока, что, вероятно, связано с резким изменением электронной проводимости за счет неклассических механизмов транспорта электронов.

\section{Конфликт интересов}

Авторы заявляют, что у них нет конфликта интересов.

\section{Список литературы}

[1] Kim V. // Journal of Propul. Power. 1998. Vol. 14. N 5. P. 736743. Doi: $10.2514 / 2.5335$

[2] Morozov A.I., Savelyev V.V. Reviews of Plasma Physics. / Ed. by B.B. Kadomtsev, V.D. Shafranov. NY.: Consultant Bureau, 2000. Vol. 21. P. 203-391.

[3] Mikellides I.G., Katz I., Hofer R.R., Goebel D.M. // J. Appl. Phys. 2014. Vol. 115. N 4. P. 043303.

[4] Hofer R.R., Goebel D.M., Mikellides I.G., Katz I. // J. Appl. Phys. 2014. Vol. 115. N 4. P. 043304.

[5] Zakharenkov L.E., Kim V., Lovtsov A.S., Semenkin A.V., Solodukhin A.E. // Proc. of the Space Propulsion. 2018 Conference. Seville, Spain, 2018. SP2018_paper 00195.

[6] Semenkin A.V. // Proc. of the 23th International Electric Propulsion Conference. Cleaveland, USA, 1993. Paper IEPC-93-231.

[7] Semenkin A. etal. // Proc. 25 ${ }^{\text {th }}$ International Electric Propulsion Conference. Seattle, USA, 1997. Paper IEPC-97-106.

[8] Conversano R.W., Goebel D.M. etal. // Proc. of the $50^{\text {th }}$ AIAA/ASME/SAE/ASEE Joint Propulsion Conference. Cleveland, USA, 2014. DOI: 10.2514/6.2014-3896

[9] Sekerak M.J. Plasma Oscillations and Operational Modes in Hall Effect Thrusters. PhD thesis. University of Michigan, 2014.

[10] Hara K., Sekerak M.J., Boyd I.D., Gallimore A.D. // J. Appl. Phys. 2014. Vol. 115. P. 203304.

[11] Ding Y., Boyang J. etal. // Adv. Space Res. 2018. Vol. 61. N 3. P. 837-843. https://doi.org/10.1016/j.asr.2017.11.003

[12] Ding Y., Sun H. etal. // Vacuum. 2017. Vol. 143. P. 251-261. http://dx.doi.org/10.1016/j.vacuum.2017.06.030

[13] Han L., Wei L., Yu D. // J. Phys. D: Appl. Phys. 2016. Vol. 49. P. 375203.

[14] Azziz Y. Experimental and theoretical characterization of a Hall thruster plume. PhD thesis. Massachusetts Institute of Technology, 2007.

[15] Ловиов А.С., Томилин Д.А., Шашков А.С. // Письма в ЖТФ. 2014. Т. 40. Вып. 17. С. 60-68. [Lovtsov A.S., Tomilin D.A., Shashkov A.S. // Tech. Phys. Lett. 2014. Vol. 40. N 9. P. 754-757.

[16] Kostin A.N. etal. // Proc. of the $33^{\text {th }}$ International Electric Propulsion Conference. Washington, USA, 2013. Paper IEPC-2013-055.

[17] Горшков О.А., Шагайда А.А. // Письма в ЖТФ. 2008. Т. 34. Вып. 4. С. 37-43. [Gorshkov O.A., Shagaida A.A. // Tech. Phys. Lett. 2008. Vol. 34. N 2. P. 153-155.]

[18] Shagayda A.A. // IEEE Tr. Plasma Sci. 2015. Vol. 43. N 1. P. 12-28. Doi: 10.1109/TPS.2014.2315851

[19] Nikitin V., Tomilin D., Lovtsov A., Tarasov A. // EPL. 2017. Vol. 117. N 4. P. 45001.

https://doi.org/10.1209/0295-5075/117/45001 